\title{
Multiple Sites of Adaptive Plasticity in the Owl's Auditory Localization Pathway
}

\author{
William M. DeBello ${ }^{1,2}$ and Eric I. Knudsen ${ }^{1}$ \\ ${ }^{1}$ Department of Neurobiology, Stanford University School of Medicine, Stanford, California 94305-5125, and 2Center for Neuroscience, Department of \\ Neurobiology, Physiology and Behavior, University of California-Davis, Davis, California 95616
}

In the midbrain auditory localization pathway of the barn owl, a map of auditory space is relayed from the external nucleus of the inferior colliculus (ICX) to the deep and intermediate layers of the optic tectum (OT) and from these layers to the superficial layers. Within the 0T, the auditory space map aligns with a visual map of space. Raising young barn owls with a prismatic displacement of the visual field leads to progressive changes in auditory tuning in the OT that tend to realign the auditory space map with the prismatically displaced visual space map. The only known site of this adaptive plasticity is in the ICX, in which the auditory system first creates a map of space. In this study, we identified an additional site of plasticity in the OT. In owls that experienced prisms beginning late in the juvenile period, adaptive shifts in auditory tuning in the superficial layers of the 0T exceeded the adaptive shifts that occurred in the deep layers of the OT or in the ICX. Anatomical results from these owls demonstrated that the topography of intrinsic 0T connections was systematically altered in the adaptive direction. In juvenile owls, plasticity in the OT increased as plasticity in the ICX decreased. Because plasticity at both sites has been shown to decline substantially in adults, these results suggest that an age-dependent decrease in auditory map plasticity occurs first in the ICX and later at the higher level, in the OT.

Key words: binaural cues; experience; juvenile; prisms; retrograde labeling; sensitive period

\section{Introduction}

Experience can cause plasticity at various levels in neural networks (Daw et al., 1992; Raymond et al., 1996; Gilbert, 1998; Jones, 2000). Identification of sites of plasticity is an essential first step to understanding the mechanisms by which experience shapes the functional properties of the brain. The midbrain auditory localization pathway in barn owls is known to be highly plastic, but until now, only a single site of plasticity has been recognized (Brainard and Knudsen, 1993; Knudsen, 2002). This study identifies a second site of plasticity, one that exists at a higher level in the pathway and exhibits a different pattern of age-dependent regulation.

The barn owl midbrain auditory localization pathway extracts spatial information from auditory localization cues (Cohen and Knudsen, 1999). Binaural cues, including interaural time differences (ITDs) and interaural level differences (ILDs), are encoded in frequency-specific channels in the central nucleus of the inferior colliculus (ICC). This information is combined across frequency channels to create a map of space in the external nucleus of the inferior colliculus (ICX). The auditory map of space is relayed by topographic connections to the deep and intermediate layers of the optic tectum (OT) and, from there, to the superficial

Received Feb. 10, 2004; revised June 8, 2004; accepted June 10, 2004.

This work was supported by grants from the McKnight Foundation and by National Institutes of Health-National Institute on Deafness and Other Communication Disorders Grant R01 DC00155-23. We thank Y. Gutfreund, K. Maczko, and J. Bergan for comments on this manuscript.

Correspondence should be addressed to W. M. DeBello, Center for Neuroscience, Department of Neurobiology, Physiology and Behavior, University of California-Davis, Davis, CA 95616.

DOI:10.1523/JNEUROSCI.0480-04.2004

Copyright $\odot 2004$ Society for Neuroscience $\quad$ 0270-6474/04/246853-09\$15.00/0 layers (Knudsen and Knudsen, 1983). In the OT, the auditory map is aligned and merged with a visual space map to create a multimodal space map (Knudsen, 1982).

The representation of auditory cues in the midbrain pathway is shaped by experience during early life (Knudsen, 1999). For example, in juvenile barn owls raised with prismatic spectacles (prisms) that displace the visual field horizontally, neurons in the ICX and OT change their spatial tuning in a way that tends to align the auditory space map with the prismatically displaced representation of visual space (Knudsen and Brainard, 1991). This change is attributable primarily to a shift in the tuning of neurons to ITDs (Brainard and Knudsen, 1993), the primary cue for the horizontal position of a sound.

The ICX has been identified as a major site of this adaptive plasticity. Experience-driven changes in the auditory space map are associated with corresponding changes in the topography of axonal projections from the ICC to the ICX (Feldman and Knudsen, 1997; DeBello et al., 2001) and with changes in patterns of both excitation and inhibition within the ICX (Zheng and Knudsen, 1999; Zheng and Knudsen, 2001).

The superficial layers of the OT (superior colliculus in mammals) have been identified as providing critical, instructive information for the developmental emergence [ferrets (King et al., 1998)] and adaptive adjustment [barn owls (Hyde and Knudsen, 2002)] of the auditory space map. Here we demonstrate that the OT can also serve as a site of auditory map plasticity. Previous research focused on plasticity in early juvenile owls (Knudsen, 1999), typically $<70$ d old (barn owls reach sexual maturity at 200-250 d old). In such young owls, functional changes in the OT simply reflect plasticity that takes place in the ICX. The 
present study includes older juveniles and reveals an independent capacity for plasticity resident to $\mathrm{OT}$.

\section{Materials and Methods}

Experiments were conducted on a total of 28 barn owls (Tyto alba). All procedures were approved by the Stanford University Institutional Animal Care and Use Committee.

Prism rearing. For surgical attachment of headgear, owls were anesthetized with 1.5\% halothane in nitrous oxide/oxygen (4:5), the scalp was cleaned with betadine solution, and the skull was exposed. A mount for the prisms was cemented to the front of the skull with dental acrylic, and a small plate for securing the head in the recording apparatus was cemented to the back of the skull. Surgical incisions were treated with betadine, sutured, and infused with lidocaine hydrochloride. Owl Ringer's solution ( $2 \mathrm{cc} ; 2.5 \%$ dextrose in $0.75 \%$ saline) was administered via intramuscular injection, and the owl was allowed to recover overnight before being released into an aviary. At various ages, prismatic spectacles (prisms) that shifted the visual field by $23^{\circ}$ either to the right $\left(\mathrm{R} 23^{\circ}\right)$ or the left $\left(\mathrm{L} 23^{\circ}\right)$ were mounted. The owls were released into large aviaries with other owls that provided a rich environment to induce adaptive adjustment to the prisms. The duration of prism experience in these aviaries ranged from 1 week to several months.

Acute recordings. Twenty-four owls underwent from one to seven acute recording sessions spaced no less than 1 week apart. For each session, the owl was anesthetized with halothane, placed in a restraining device, and secured in the stereotaxic recording apparatus. Craniotomies were opened on the basis of stereotaxic coordinates. Owls were maintained under nitrous oxide/oxygen (4:5), and halothane was applied briefly only if an owl became active. Epoxylite-coated tungsten electrodes (0.3-2.0 M $\Omega$ ) were lowered through the forebrain into the structures of interest. The surface of the brain was periodically irrigated with chloramphenicol solution $(0.5 \%)$. At the end of a recording session, the craniotomies were infused with chloroptic ointment $(1 \%)$ and sealed with dental acrylic, and the owl was placed in a recovery box overnight and returned to its aviary the next day.

Chronic recordings. Long-term recordings were made from matched sites in the ICX, deep OT, and superficial OT in four owls. Electrodes were implanted at sites representing a single region of space, as indicated by overlapping ITD tuning (within $\pm 10 \mu \mathrm{sec}$ ) and visual receptive fields $(\mathrm{Vrfs})\left(\right.$ within $\left.4^{\circ}\right)$. Electrodes were cemented to the skull, bent parallel to the surface, and embedded in dental acrylic. Contact points were established by sanding the dental acrylic, exposing a portion of each electrode. For recording, connection to a preamplifier was made by lowering a small stainless steel tube onto the exposed contact.

Prisms were mounted within a few days of electrode implantation. Measurements were made during short recording sessions $(<4 \mathrm{hr})$, spaced by $\sim 1$ week, and repeated as long as high quality recordings could be obtained (up to 8 weeks). For the sites included in this report, the measured Vrfs did not change by $>2^{\circ}$ across any of the sessions. The stability of the Vrfs across months of prism experience is consistent with previous reports (Brainard and Knudsen, 1995) and indicates that there was minimal change in the position of the electrode in the brain.

Visual and auditory measurements. All data were based on multiunit responses. Vrfs were assessed with the prisms removed temporarily. They were measured by projecting positive or negative contrast bars and spots onto a calibrated tangent screen and listening to the unit responses broadcast over an audiomonitor. The geometric center of the Vrf was defined as the best Vrf.

Acoustic stimulation and data acquisition were controlled by the SOUND program (written by J. Gold, University of Pennsylvania). Acoustic stimuli were presented dichotically through earphones
B

C

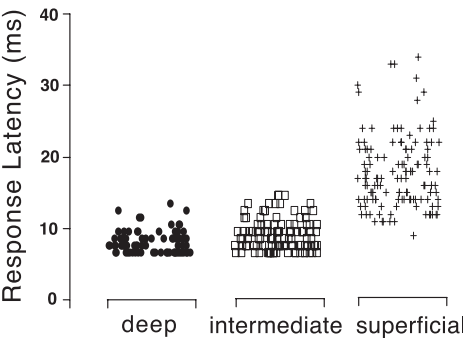
were calibrated with Bruel and Kjaer condenser microphones and a spectrum analyzer. Stimulus waveforms were adjusted to equalize the amplitude and phase spectra of the earphones to within $\pm 2 \mathrm{~dB}$ and $\pm 2 \mu \mathrm{sec}$, respectively, over the frequency range of $2-10 \mathrm{kHz}$. Earphones were placed in the ear canals $\sim 5 \mathrm{~mm}$ from the tympanic membrane. Auditory stimuli were $3 \mathrm{kHz}$ high-pass-filtered noise bursts, $50 \mathrm{msec}$ in duration, 0 $\mathrm{msec}$ rise-fall times, presented once per second, at an average binaural level of $20 \mathrm{~dB}$ above unit threshold.

A level discriminator was used to isolate action potentials generated by a small number of neurons at each recording site, and the spike times were stored. For deep and superficial layer sites, response magnitude was defined as the number of action potentials occurring during the $50 \mathrm{msec}$ sound stimulus minus the number occurring in the $50 \mathrm{msec}$ preceding the stimulus. For intermediate layer sites, response magnitude was calculated separately for two separate epochs, $0-18$ and $18-50 \mathrm{msec}$ after stimulus (see Results for explanation), and a prorated amount of baseline activity was subtracted.

ITD tuning was measured by presenting noise bursts with ITDs that varied in $10 \mu \mathrm{sec}$ increments across the response range of the site. Rightear-leading ITDs are designated as positive values, and left-ear-leading ITDs are designed as negative values. Stimulus series were presented $10-50$ times, with ITDs varied in a random interleaved order, and at the best ILD for the site. The best ILD was determined at an ITD within \pm 10 $\mu$ sec of the best ITD.

ITD tuning (best ITD) was quantified as the weighted average of unit responses as a function of ITD. Shifts in best ITD tuning were quantified by comparing the measured ITD tuning with the ITD tuning predicted from the location of the best Vrf (normal ITD tuning). The predicted normal ITD tuning was calculated according to the following formula: predicted normal ITD $=2.5 *$ azimuth of best Vrf. This relationship holds for tectal locations representing frontal space at or below the horizon (Brainard and Knudsen, 1993). Data collection was restricted to sites within this region. Shift in ITD tuning was calculated as follows: weighted average of measured ITD tuning - predicted normal ITD.

Identification of recording sites. The avian OT contains 15 layers. These layers have been grouped previously into superficial (layers 1-10) and deep (11-15) on the basis of differences in anatomical connections and patterns of electrophysiological responses (Knudsen et al., 1982; Reiner and Karten, 1982; Knudsen and Knudsen, 1983). For the purposes of this study we have subdivided the deeper layers into intermediate (layers 11-12) and deep (layers 13-15).

Multiunit recording sites were assigned to specific tectal layers on the basis of the following criteria. Sites in the superficial layers responded at relatively long latencies to auditory stimulation (Fig. 1C), and they 
A

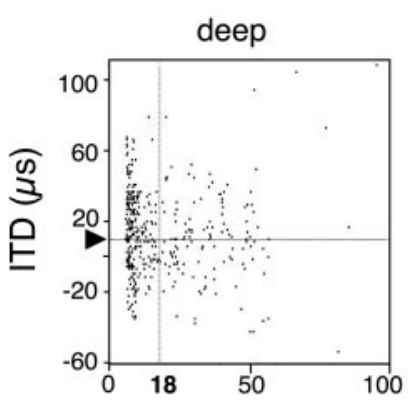

B
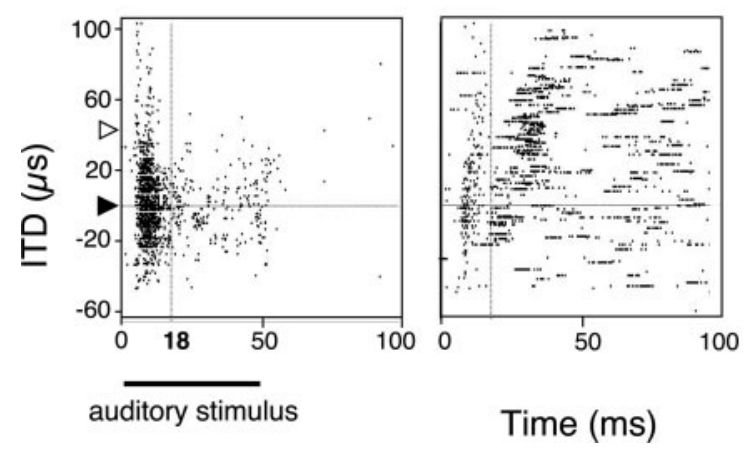

Figure 2. Layer-specific response patterns and alignment of ITD tuning across the tectal layers. Raster plots of auditory responses in deep (left), intermediate (middle), and superficial (right) layers. ITD of the test stimulus is shown on the ordinate; peristimulus time is shown on the abscissa. Duration of the auditory stimulus is indicated by the bar. Each dot represents the time of occurrence of an action potential. $A$, Normal owl. Horizontal line corresponds to the best ITD predicted from the observed Vrf ( $R 4^{\circ}$ for all). Responses in the deep layers were regular in discharge pattern, occurred with short latencies, and essentially ceased by $18 \mathrm{msec}$ after stimulus onset (vertical line). Responses in superficial layers (right panel) discharged in bursts, always had longer onset latencies, and usually were strongest $\geq 18 \mathrm{msec}$ after the stimulus. Regardless of discharge pattern, ITD tuning was very similar at all three sites (see Results for details). B, Prism-reared owl. The open arrowhead (instructed) corresponds to the best ITD predicted by the prismatically displaced Vrf; the filled arrowhead (normal) corresponds to the ITD predicted by the Vrf with prisms removed ( $\mathrm{Vrf}=0^{\circ}$ for all). In this owl, activity in the deep layers and short-latency activity in the intermediate layers had shifted minimally toward the instructed value of ITD. In contrast, long-latency activity in the intermediate and superficial layers had shifted substantially toward the instructed value. The temporal response patterns in each layer were similar to those observed in normal owls.

tended to discharge in bursts. Sites in the deep layers responded at short latencies (Fig. 1C) and with a Poisson (regular) pattern of discharge. Sites in the intermediate layers exhibited the properties of both superficial (discharging in bursts) and deep (regular discharges at short latency) units. Because waveform analysis was not performed, it was not possible to determine whether individual units gave rise to both response patterns. These layer assignments were validated by reconstructing electrolytic lesion made in this study (Fig. $1 B$ ) and previous studies (Knudsen, 1982).

Subdivisions of the IC were identified on the basis of characteristic patterns of tuning to ITD, ILD, and frequency (Feldman and Knudsen, 1994).

ICC-OT transects. To assess shifts in ITD tuning in the ICX, where units do not normally exhibit Vrfs (Gutfreund et al., 2002), recordings were made along transects connecting the representation of $0 \mu \mathrm{sec}$ in the ICC, with the representation of $0^{\circ}$ azimuth in the OT. Because prism rearing alters neither the representation of ITDs in the ICC nor that of visual space in the OT (Brainard and Knudsen, 1993; Feldman and Knudsen, 1997), this transect passes through the same anatomical locations in the ICX in normal and prism-reared owls. In normal owls, these locations are all tuned to $0 \mu \mathrm{sec}$ ITD.

Retrograde labeling and analysis. Injections of biotinylated dextranamine (BDA) in the OT were centered in layer 10 at a consistent, rostrocaudal location: at the representation of contralateral ear-leading 15 $\mu$ sec. A $5 \%$ solution of BDA in $0.76 \%$ saline was freshly prepared and loaded into a thin-walled, fiber-filled borosilicate glass pipette $(1.5 \mathrm{~mm})$ with an inner tip diameter of $15 \mu \mathrm{m}$. Multiunit activity was used to position the tip of the pipette in layer 10 . BDA was ejected by passing $3 \mu \mathrm{A}$ of positive current with a $50 \%$ duty cycle, $7 \mathrm{sec}$ on/off, for $15 \mathrm{~min}$. The electrode was left in place for 15 min and then slowly withdrawn to the top of the OT.

After 3-4 d of survival, the owl was deeply anesthetized with 5\% halothane and nitrous oxide/oxygen (4:5). The thoracic cavity was opened and $0.5 \mathrm{cc}$ of Nembutal was injected into the liver. Heparin (300 U) was injected into the left ventricle, and the owl was perfused transcardially with $500 \mathrm{ml}$ of $0.1 \mathrm{M}$ phosphate buffer (PB) containing lidocaine $(3 \mathrm{ml} / \mathrm{l})$, followed by $500 \mathrm{ml}$ of $4 \%$ paraformaldehyde in $\mathrm{PB}$, followed by $200 \mathrm{ml}$ of $4 \%$ paraformaldehyde in PB with $10 \%$ sucrose. The brain was removed and sunk in $4 \%$ paraformaldehyde in PB with 30\% sucrose. After 3-7 d, $40 \mu \mathrm{m}$ sections were cut though the midbrain in the horizontal plane defined by the long axis of the OT. Sections were cut on a freezing microtome and stored at $4^{\circ}$ overnight in $\mathrm{PB}$. Sections were reacted with the ABC method (Vector Laboratories, Burlingame, CA), lightly counterstained with cresyl violet, dehydrated, cleared in xylenes, and coverslipped with Permount.

The spatial patterns of BDA-labeled somata were assessed by a person who was blind to the experiment. Image acquisition and analysis were performed with a Nikon Eclipse E800 microscope, a SPOT digital camera (Diagnostic Instruments, Sterling Heights, MI), and Simple32 image processing software (Compix, Lake Oswego, OR). For each image, the rostrocaudal location of the center of the injection site was marked with a line running perpendicular to the layers of OT. The distance of retrogradely labeled somata from this line was measured, and the layer was noted. All measurements were pooled across sections aligned on the injection site. A cumulative frequency distribution was computed for each case (three normal, six prismreared), and the rostrocaudal locations, relative to the injection site, where cumulative frequencies reached $50 \%$ (the location of the median labeled cell) were determined.

The spatial distributions of labeled somata were also evaluated for symmetry relative to the site of injection as follows. For each $100 \mu \mathrm{m}$ interval for which labeled cells were observed on both sides of the injection site, the difference between cell counts at symmetrical distances from the injection site was normalized by total number of cells for that distance. The distribution of these normalized values was tested for deviation from perfect symmetry (zero) with a two-tailed Student's $t$ test.

\section{Results}

\section{Layer-specific ITD maps in the optic tectum.}

Normally, maps of ITD are mutually aligned across the layers of the OT. This was not the case for 16 owls that began wearing displacing prisms late in the juvenile period.

Examples of the patterns of responses and the mutual alignment of ITD tuning that were observed routinely across the tectal layers in normal owls are shown in Figure $2 \mathrm{~A}$. For these data, visual receptive fields were centered at $\mathrm{R} 4^{\circ}$, which predicts the best ITDs of $+10 \mu \mathrm{sec}$ (see Materials and Methods). The deep layer site (Fig. 2A, left panel) responded at short latency ( $7 \mathrm{msec})$ 
and was tuned to $+8 \mu \mathrm{sec}$ ITD. The intermediate layer site (middle panel) also responded at short latency $(7 \mathrm{msec})$ and was tuned to $+11 \mu \mathrm{sec}$ ITD. The superficial layer site (right panel) responded at long latency $(15 \mathrm{msec})$ with bursts of action potentials and was tuned to $+10 \mu \mathrm{sec}$ ITD.

The pattern of ITD tuning across the layers of the OT was highly abnormal in a juvenile owl that had experienced prisms for 8 weeks beginning at $120 \mathrm{~d}$ old, as shown in Figure $2 B$. For these data, visual receptive fields were centered at $0^{\circ}$ azimuth, which predicts the best ITDs of $0 \mu \mathrm{sec}$. Response latencies and discharge patterns were similar to those observed in the normal owl (Fig. $2 \mathrm{~A}$ ); however, the best ITD for the deep layer site (left panel) was shifted by $5 \mu$ sec relative to the predicted normal value. The shift was in the adaptive direction, compensating for the leftwardshifting prisms. For the intermediate layer site (middle panel), short- and long-latency responses were tuned to different values of ITD. The change in ITD tuning appeared $\sim 18 \mathrm{msec}$ after stimulus onset, based on visual inspection of the data. Therefore, best ITDs were evaluated separately for responses occurring $<$ or $>18$ msec after stimulus onset (Fig. 2, vertical lines). The best ITD for the short-latency responses was $+6 \mu \mathrm{sec}$, and for the long-latency responses it was $+32 \mu \mathrm{sec}$. For responses that began $>18 \mathrm{msec}$ after stimulus onset, latencies to learned values of ITD were longer than responses to normal ranges of ITD (Fig. $2 B$, middle panel). At the superficial layer site (Fig. $2 B$, right panel), the best ITD was $+29 \mu$ sec. As has been reported previously (Brainard and Knudsen, 1995), units in the superficial OT responded with longer latencies to learned versus normal ranges of ITD.

Data such as those shown in Figure $2 B$ indicate a shift in the map of ITD across the layers of the OT. The magnitude of this shift was quantified by comparing ITD tuning in the deep layers with ITD tuning in the superficial layers and by comparing the ITD tuning of short-latency responses in the intermediate layers with that of long-latency responses in the intermediate layers.

\section{Comparison of ITD maps in the deep and superficial layers}

The magnitudes of the shifts in the ITD maps in the deep versus superficial layers were measured in prism-reared owls after at least 8 weeks of prism experience [previous studies have demonstrated that most of the adjustment in ITD tuning occurs after $<8$ weeks of prism experience (Brainard and Knudsen, 1998)]. Shifts from predicted normal ITD tuning were measured at $5-18$ sites in the superficial and deep layers, and the data from each set of layers were averaged to yield population ITD tuning curves. The difference between the weighted averages of these population tuning curves was used as a metric for the magnitude of the cross-layer ITD tuning shift in the OT of that animal. All recording sites were from the portion of the OT representing frontal space (best $\mathrm{Vrfs}$ between $\mathrm{L} 15^{\circ}$ and $\mathrm{R} 15^{\circ}$ azimuth and between $+10^{\circ}$ and $-20^{\circ}$ elevation), where prism experience has been shown to cause uniform shifts in the ITD map (Knudsen, 1998).

Alignment of the superficial ITD map relative to the deep map is assessed in Figure 3 for three representative owls. On the left are shown ITD tuning curves from individual sites in the superficial and deep layers, respectively, recorded in the visual representation of $0^{\circ}$ azimuth, where sites are normally tuned to $0 \mu$ sec ITD. On the right are population tuning curves summarizing all data collected from that owl. In the normal owl, ITD tuning was aligned across the superficial and deep layers and centered on the predicted normal value (Fig. $3 A, B$ ). In the owl that experienced prisms for 8 weeks beginning at $75 \mathrm{~d}$ old, ITD tuning was also aligned across the superficial and deep layers, but was shifted from normal by an average of $\sim 40 \mu \mathrm{sec}$ in the adaptive direction
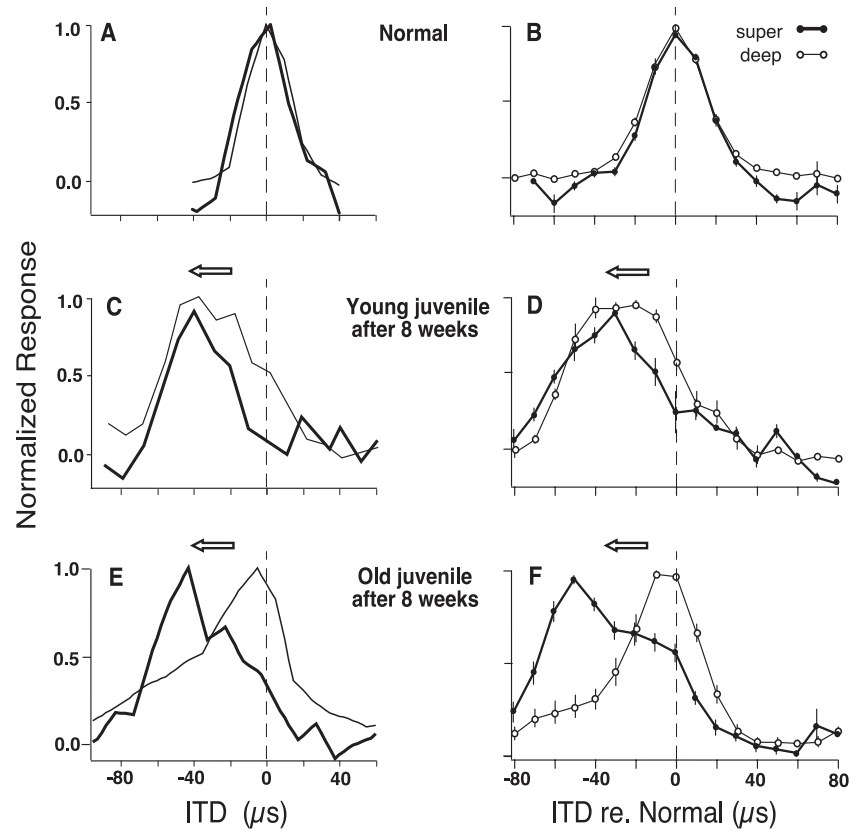

Figure 3. Comparison of ITD tuning in the deep and superficial layers in owls with 8 or more weeks of prism experience. Single site (left panels) and population tuning curves (right panels) from a normal owl $(A, B)$, an owl in which prisms were mounted as a young juvenile, at $75 \mathrm{~d}(C$, $D)$, and an owl in which prisms were mounted as an older juvenile, at $120 \mathrm{~d}(E, F)$, are shown. Tuning curves with thin lines and open symbols were recorded in deep layers; those with thick lines and filled symbols were recorded in superficial layers. The Vrfs for sites in $A, C$, and $E$ were at $0^{\circ}$ azimuth. The population tuning curves are aligned relative to the predicted normal ITD (dashed vertical line). The adaptive direction is shown to the left. Error bars are SEM. ITD maps in deep and superficial layers were well aligned in normal owls and in owls for which prisms were mounted as young juveniles. In contrast, the ITD maps in older juveniles were misaligned; the amount of adaptive shift in superficial layers often exceeded that in the deep layers. The reverse was not observed.

A

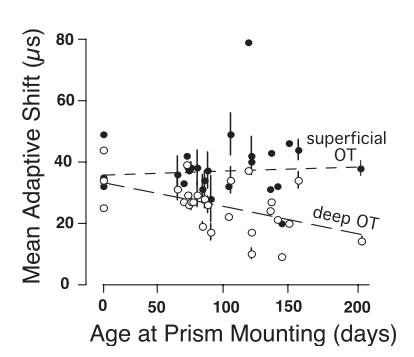

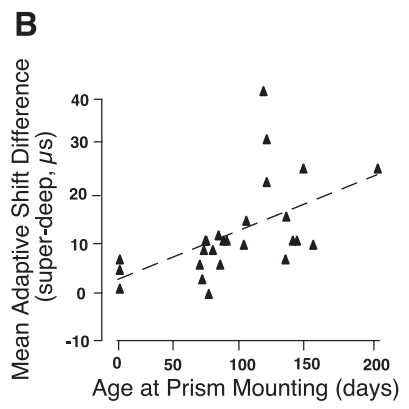

Figure 4. Age dependence of cross-layer plasticity: deep versus superficial layers. The mean adaptive shift for owls with $>8$ weeks of prism experience was calculated separately for the superficial and deep layers. Each point represents all data from one owl. $A$, The amount of plasticity expressed in the deep layers (open symbols) declined with age of prism mounting (linear regression; $p<0.005$ ) but was independent of age in the superficial layers (filled symbols; $p>0.05$ ). Error bars are SEM. $B$, Difference in mean adaptive shift between superficial and deep layers increased with age of prism mounting $(p<0.005)$.

(Fig. 3C,D). In the owl that experienced prisms for 8 weeks beginning at $120 \mathrm{~d}$ old, ITD tuning was not aligned across OT layers (Fig. $3 E, F$ ). ITD tuning at sites in the deep layers was shifted from normal by an average of $10 \mu \mathrm{sec}$, whereas ITD tuning at sites in the superficial layers was shifted by an average of $32 \mu \mathrm{sec}$. This corresponds to an ITD map misalignment between the superficial and deep layers of $22 \mu$ sec.

Superficial versus deep layer map alignments, assessed in the manner shown in Figure 3, are summarized in Figure 4 for all 
A

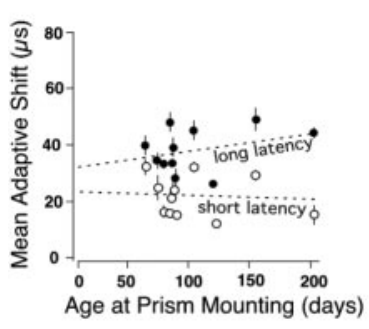

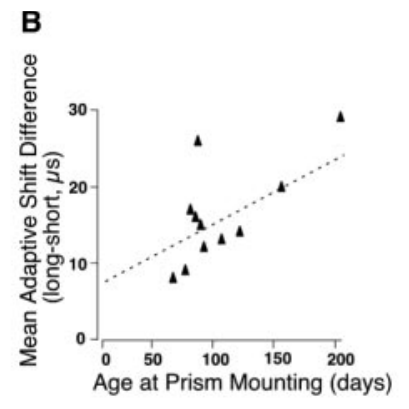

C

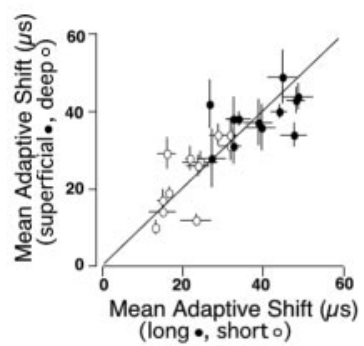

Figure 5. Age dependence of intermediate layer plasticity: short versus long latency. The mean adaptive shift for owls with $>8$ weeks of prism experience was calculated separately for short- and long-latency activity recorded at intermediate layer sites. Each point represents all data from one owl. A, Plasticity expressed by short-latency responses (open symbols); plasticity expressed by the long-latency responses (filled symbols). $B$, For data measured in the same animal, differences in mean adaptive shift between short- and long-latency activity increased with age of prism mounting. C, For data from individual animals, magnitudes of shift in tuning for activity in the superficial layers covaried with those for long-latency activity from the intermediate layers (filled symbols), as well as the magnitudes of shift in tuning for activity from the deep layers covaried with those for short-latency activity in the intermediate layers (open symbols). All error bars are SEM.

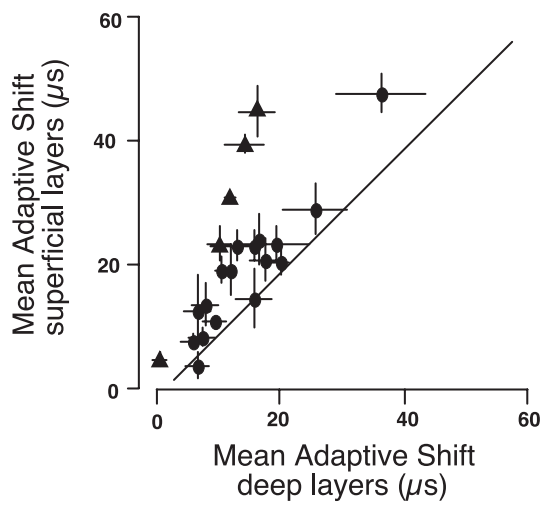

Figure 6. Comparison of shifts in ITD tuning in the deep versus superficial layers in owls with $<8$ weeks of prism experience. Recordings were made in a subset of owls that were actively adjusting to prisms. Each point represents one recording session $(n=22)$. The solid line represents equal adjustment of ITD tuning in the superficial and deep layers. Filled triangles represent significant differences between the layers (unpaired $t$ test; $p<0.05$ ). Filled circles represent nonsignificant differences. All but two of the points fall above the diagonal line, indicating a strong tendency toward cross-layer splits in adjusting owls (paired $t$ test; $p<0.01$ ). Error bars are SEM.

owls in this study. The data from the experimental owls were gathered after the owls had experienced prisms for at least 8 weeks, the duration beyond which prism experience causes little additional plasticity in juvenile owls (Brainard and Knudsen, 1998). Figure $4 A$ shows the average shift of ITD tuning relative to normal for the superficial and deep layers, respectively. Among the experimental owls, the ITD map shift in the superficial OT did not change as a function of the age at prism mounting $(p>$ $\left.0.5 ; r^{2}=0.088 ; n=27\right)$. In contrast, the ITD map shift in the deep layers tended to decrease with age at prism mounting over this period $\left(p<0.005 ; r^{2}=0.52 ; n=27\right)$. Figure $4 B$ shows the cross-layer map misalignment calculated from the differences between these values for each owl. As owls aged during the juvenile period, cross-layer map misalignment tended to increase $\left(p<0.005 ; r^{2}=0.556 ; n=27\right)$.

In addition to shifts in tuning, prism experience caused tuning curves to broaden. Across all normal owls, the average width of ITD tuning curves (measured at half-height of each curve) was $32 \pm 1.3 \mu \mathrm{sec}(n=78)$ in the superficial layers and $33 \pm 0.9 \mu \mathrm{sec}$ $(n=63)$ in the deep layers. After prism experience, the average

width was $40.5 \pm 1.8 \mu \sec (n=189)$ in the superficial layers and $43.2 \pm 1.5 \mu \mathrm{sec}(n=$ $119)$ in the deep layers. The experienceinduced broadening was significant for both layers $(t$ test; $p<0.001)$. The increased breadth of tuning was caused by the coexpression of responses to both normal and adaptive values of ITDs (Fig. 3, compare $C$ or $E, A$ ).

\section{Comparison of short- and long-latency ITD maps in the intermediate layers}

In owls that experienced prisms for 8 weeks beginning after $80 \mathrm{~d}$ old, the ITD tuning of short- and long-latency responses tended to differ (Fig. 2B, middle panel). Because the change in ITD tuning appeared at $\sim 18 \mathrm{msec}$ after stimulus onset, tuning was assessed separately for intermediate layer responses occurring $0-18$ versus $18-50 \mathrm{msec}$ after stimulus onset. Figure 5 summarizes the data for 11 owls. Among the experimental owls, the average shift from normal of ITD tuning for long-latency responses was always larger than the average shift from normal for short-latency responses. The differences between the shifts in ITD tuning for the long- versus short-latency responses measured in individual birds are shown in Figure $5 B$. These differences tended to increase with age during the juvenile period $\left(p<0.005 ; r^{2}=0.74 ; n=11\right)$. Moreover, in data from individual owls, the magnitude of the shifts in ITD tuning measured for long-latency responses in the intermediate layers covaried with the magnitude of the shifts measured in the superficial layers (Fig. 5C, filled symbols), and those of short-latency responses in the intermediate layers covaried with the magnitude of the shifts measured in the deep layers (Fig. 5C, open symbols).

\section{Dynamics of plasticity}

The results in the previous sections were obtained from owls in a steady state of shift, after at least 8 weeks of prism experience. Cross-layer map plasticity was also assessed earlier in the adjustment process, as the changes were taking place. These recordings were made after 7-40 d of prism experience in eight owls. Average shifts of ITD tuning from normal for all deep and all superficial layer sites were calculated and compared (Fig. 6). Average shifts in ITD tuning in the deep layers ranged from -1 to $34 \mu$ sec; in the superficial layers, they ranged from 5 to $48 \mu \mathrm{sec}$. The sizes of the shifts increased with the amount of experience (data not shown). During this period, whenever there was a difference between the ITD maps, the shift of the ITD map in the superficial layers was always larger than the shift in the deep layers.

Chronic recording techniques were used to follow the time course of the adaptive changes (Fig. 7). Electrodes were implanted in four owls at sites in the superficial OT, deep OT, and ICX that exhibited similar ITD and ILD tuning. We selected older juveniles, aged $85-155 \mathrm{~d}$, to increase the likelihood that adjustments would occur at different rates in the deep versus superficial layers.

Adaptive changes in ITD tuning appeared within $7 \mathrm{~d}$ after the beginning of prism experience. The shift in the superficial layers was larger than the shift in the deep layers in all cases (Fig. 7A-D), indicating plasticity in the OT (Fig. 7E). Most of the OT plasticity took place in the first 3 weeks of prism experience for two cases (Fig. $7 A, C, E$ ), and in only one case did OT plasticity continue for the duration of the experiment (Fig. $7 B, E$ ). 


\section{Adaptive plasticity of anatomical connections}

The additional shift in the ITD map that appeared between the deep and superficial OT could have been caused by a change in intratectal connections. For rostralward cross-layer map shifts, the predicted anatomical change would be a caudalward shift in retrogradely labeled cell bodies in the OT, and for caudalward map shifts, a rostralward shift in retrogardely labeled cells. To test this hypothesis, small injections, centered in layer 10 , of the retrograde tracer $\mathrm{BDA}$ were made into three normal tecta and six tecta that exhibited different degrees of ITD map misalignment across the deep and superficial layers (Fig. 8). The anatomical data were analyzed (see Materials and Methods) by an observer who was blind to the experiment.

In the normal tecta, retrograde labeling was symmetrical around the injection site along the rostrocaudal dimension in both the superficial and deeper layers (Fig. $9 A, D$ ) (two-tailed Student's $t$ test; $p>$ 0.01). Cumulative frequency analysis (data not shown) indicated that the centers of the distributions of labeled cells were located an average of 49 and $48 \mu \mathrm{m}$ caudal to the injection site in the superficial and deeper layers, respectively.

In contrast, in four of the six tecta that exhibited cross-layer map misalignments, the rostrocaudal distributions of retrogradely labeled somata in the superficial or deeper layers were shifted relative to the normal range (Fig. $9 B, C, E, F$, curves marked with filled symbols) $(p<.01)$. The direction of the anatomical shifts was always in the direction predicted by the prisms for that side of the brain (Fig. 10). There was no correlation between the size of the cross-layer ITD map shift and the size of the anatomical shift.

\section{Discussion}

These experiments demonstrate that the OT is a site of plasticity in the owl's midbrain auditory localization pathway. Like the plasticity that occurs in the ICX, the plasticity in the OT is developmentally regulated: the same conditions that induce dramatic auditory map plasticity in the OT of older juveniles induce little plasticity in adults (Brainard and Knudsen, 1998); however, the age dependence of OT plasticity differs from that of ICX plasticity. Beyond $80 \mathrm{~d}$ old, ICX plasticity declines, whereas the capacity for plastic change in the OT continues (Figs. 4, 5) until $\sim 200 \mathrm{~d}$ and declines thereafter (Brainard and Knudsen, 1998). Thus, in this pathway, as in visual and somatosensory pathways in the mammalian neocortex (Singer, 1995; Glazewski and Fox, 1996; Gilbert, 1998), the capacity for plasticity at lower levels declines before that at higher levels. A bottom-up decline of the capacity for plasticity in sensory pathways assures that lower-level representations are stable before higher-level processors commit to a particular pattern of connectivity.

A result of the bottom-up decline of plasticity in the midbrain auditory pathway is that in older juvenile owls ( $>80 \mathrm{~d}$ old) the auditory map in the ICX may adjust only slightly for the effects of the prisms, whereas the map in the superficial OT adjusts almost completely. This results in misaligned auditory maps across the layers of the OT, a highly unusual condition. In the discussion

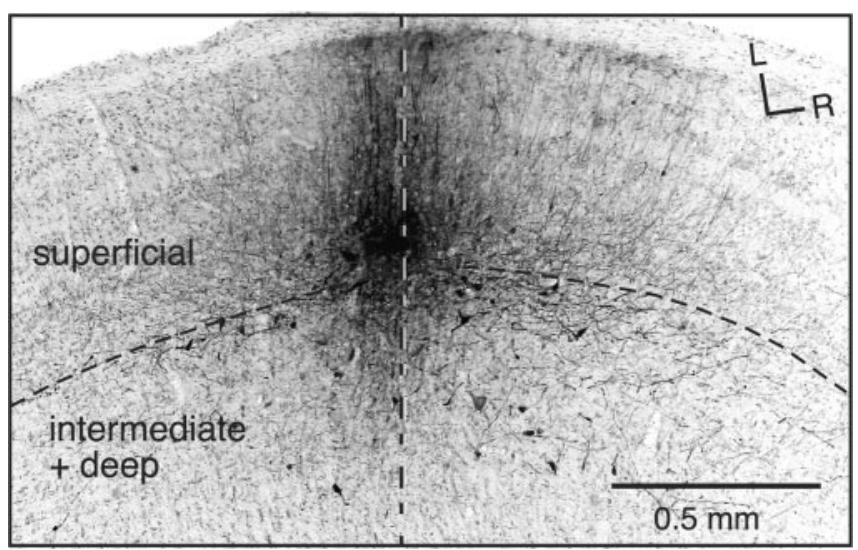

Figure 8. Retrograde labeling of intratectal connections. Horizontal section through the tectal lobe shows the location of a BDA injection site. Rostral is to the right. A light Nissl stain was applied to visualize the tectal layers. The layer 10-11 boundary is indicated with a dashed line. Numerous retrogradely labeled cell bodies were evident throughout many layers of $0 T$, superficial, lateral, and deep to the level of the injection site. The rostrocaudal level of the injection site was defined by a line through the center of the site and perpendicular the pial surface, as shown. R, Rostral; L, lateral.

that follows, we consider the mechanisms that may underlie OT plasticity and the implications of this second site of plasticity for information processing in this pathway.

\section{The OT is a site of plasticity}

Anatomical data suggest that misalignments between the auditory maps in the deep and superficial OT result, at least in part, from changes in connections within the OT (Figs. 9, 10). It is conceivable that the observed changes in the pattern of anatomical labeling in OT expressing misaligned maps (Fig. 9C,E,F) reflect changes in synaptic strength within static axonal projection fields. The other possibility is that the change in the pattern of retrograde labeling indicates the formation of new projections within the OT. In either case, the data demonstrate plasticity that 

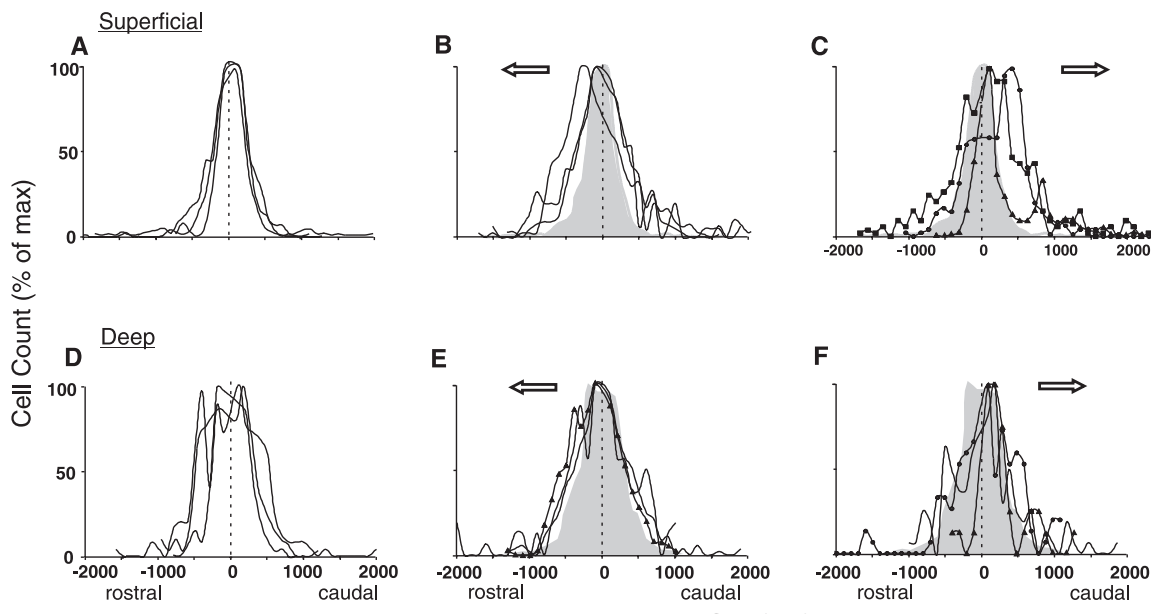

Distance from Injection Site $(\mu \mathrm{m})$

Figure 9. Altered topography of intratectal connections in normal owls and owls exhibiting cross-layer shifts in ITD tuning. Spatial distributions of retrogradely labeled cells were determined for superficial $(A-C)$ and for intermediate and deep $(D-F)$ layers. For each labeled cell, the rostrocaudal location relative to the center of the injection site was measured by a case-blind observer. Distributions were normalized by the largest number of cells labeled in a single rostrocaudal bin for each case. The spatial patterns for normal owls $(n=3)$ are shown in $A$ and $D$. The spatial patterns for owls expressing cross-layer plasticity $(n=6)$ are shown separately for cases in which there was a rostralward ITD map shift $(B, E)$ or caudalward ITD map shift $(C, F)$. The direction of ITD map shift is indicated with arrows. The composite normal distributions are shown in gray. The distributions were compared by cumulative frequency analysis (Kolomogorov-Smirnov goodness of fit for continuous data; $n>100$ ). Distributions that were different from normal $(p<0.01)$ are highlighted with filled-symbol data points.

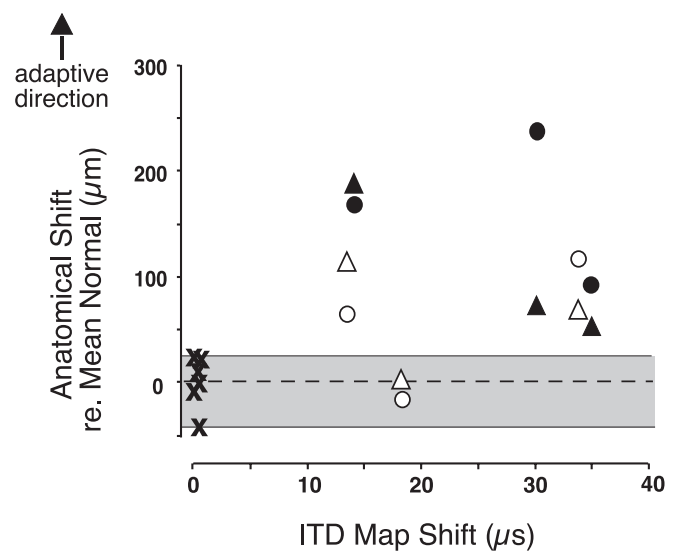

Figure 10. Summary of anatomical shifts in normal owls and owls exhibiting cross-layer shifts in ITD tuning. The anatomical shift for each case was calculated as the midpoint in the cumulative frequency curve of retrogradely filled somata (based on the data shown in Fig. 9) relative to rostrocaudal level of the injection site. This value is plotted versus the adaptive shift of the ITD map for that case. Anatomical shift for normal owls clustered near zero (crosses). The shaded region indicates the range of anatomical shifts observed in normal owls. Anatomical shifts in 10 of 12 owls exhibiting cross-layer shifts in ITD tuning fell outside the normal range: rostralward shifts (open), caudalward shifts (filled), superficial layers (circles), and deep layers (triangles). All 10 anatomical shifts were in the adaptive direction.

is intrinsic to the OT. These data do not rule out the possible contribution of additional remote sites of plasticity.

The shift in ITD tuning that occurs specifically in the longlatency responses in the intermediate layers (Figs. 2, 5) probably reflects plasticity in the superficial layers. The axons and dendrites of neurons in the superficial layers, particularly those in layers 8 and 10, extend into the intermediate layers (Ramon y Cajal, 1911; Hyde and Knudsen, 2000). The long-latency bursts recorded in the intermediate layers may represent dendritic spikes of neurons in the superficial layers or soma spikes from intermediate layer neurons that are being driven by bursting input from the superficial layers. In either of these cases, changes in the ITD tuning of long-latency responses in the intermediate layers would follow changes occurring in the superficial layers.

The latencies of responses to auditory stimulation are consistent with superficial OT plasticity being mediated by intrinsic OT connections. Previous experiments have shown that units in the ICX respond with equal latencies to normal and learned ranges of ITD (Brainard and Knudsen, 1993). In contrast, units in the superficial OT and long-latency responses in the intermediate layers occur at longer latencies to learned versus normal ranges of ITD (Brainard and Knudsen, 1995) (Fig. 2B, middle and right panels). The longer latencies of learned responses can be accounted for by learned responses being mediated by longer or additional horizontal connections within the OT. Shifted long-latency responses could reflect a synergism between deep-tosuperficial and lateral connections within the superficial layers.

The direction of both cross-layer physiological shifts and intratectal anatomical shifts was predicted by the direction of prismatic displacement. The magnitude of anatomical shift did not predict, however, the magnitude of cross-layer ITD tuning shift (Fig. 10). This lack of quantitative correspondence between anatomical and electrophysiological data could reflect owl to owl variations in local connectivity patterns that are below the resolution of the labeling technique, owl to owl variations in the strength of synapses embedded in the adaptive circuitry, or owl to owl variations in the relative contributions made by yet undiscovered sites of plasticity.

\section{Plasticity in the superficial OT}

The anatomical and electrophysiological data from this study indicate that the superficial layers of the owl's OT are a second site of plasticity in the midbrain auditory localization pathway. An adaptive advantage for auditory plasticity at such a high level in this pathway is not obvious.

The ICX, where the auditory space map is synthesized, is the obvious site for adaptive auditory plasticity to take place (Gold and Knudsen, 2000). Spatial information to the ICX arrives in frequency-tuned channels. Because growth of the head and ears, hearing loss, and changes in the properties of low-order auditory neurons alter the correspondence of encoded cue values with the locations in space in a frequency-specific manner, frequencyspecific plasticity is essential to achieve and maintain a precise representation of auditory space. As long as the plasticity in the ICX is successful in creating an auditory space map that matches a retinotopic visual template (Gutfreund et al., 2002), there is no need for further auditory plasticity at higher levels in the midbrain pathway.

In contrast, the superficial layers of the OT are not an optimal site for auditory plasticity. The auditory information that arrives at the superficial layers has already been combined across frequency channels, making frequency-specific adjustments no 
longer possible (Knudsen, 1999). Moreover, plasticity in the superficial OT results in two different auditory space maps in the OT. The superficial and deeper layers mediate different sensory and sensorimotor functions (Sprague and Meikle, 1965; Casagrande and Diamond, 1974; Stein and Meredith, 1993). One well documented function of the deep layers is the mediation of orienting movements of the eyes and head; however, neurons in the deep layers, particularly in layer 13 , project to various nuclei in the brainstem and thalamus that are involved with other less defined sensory and sensorimotor functions. The functions mediated by the superficial layers are even less well understood. The superficial layers project to the visual thalamus, the isthmo-optic nuclei, and the lateral pontine nuclei, mostly from neurons located in layers 8 and 10, suggesting various roles in sensory processing. The contribution that auditory spatial information might make to this sensory processing is not known, but the possibilities include detecting object motion and directing attention to specific locations. Evidence for the participation of the deep versus superficial layers in specific aspects of perception or behavior might be obtained by determining whether performance on behavioral tasks reflects a partially shifted (deep OT) or a fully shifted (superficial OT) map of auditory space.

The considerations listed above make layer-specific auditory map plasticity in the OT surprising; however, previous research has demonstrated an impressive capacity for activity-dependent plasticity that is intrinsic to the superficial layers of the OT in a wide range of species (Udin, 1983; Reh and Constantine-Paton, 1985; Cook and Becker, 1990; Binns and Salt, 1998). In other species, the superficial layers are exclusively visual, and this plasticity may be critical for experience-dependent adjustment of circuits involved in visual processing (Sprague and Meikle, 1965; Casagrande et al., 1972; Tunkl and Berkley, 1977; Sprague, 1991). We propose that these same mechanisms, which normally enable adaptive plasticity of visual circuits, enable auditory map plasticity in barn owls.

\section{Forces that maintain map alignment in the OT}

Despite the capacity for independent map plasticity in the superficial layers of the OT, the auditory maps in the deep and superficial layers remain aligned under most conditions; it is only in late juvenile owls that large sustained map discrepancies are observed. The tendency for the auditory maps to remain aligned across OT layers, even in owls that are in the process of adaptive adjustment, can be accounted for by the action of strong topographic feedforward projections that normally connect these layers. As long as these connections dominate over all other sources of auditory input, the map in the superficial layers will follow the map represented in the deep layers.

According to this account, the late onset of cross-layer map plasticity in the OT could be caused by the initial domination of the normal feedforward projection to the superficial layers that suppresses (perhaps through lateral inhibition) the expression of weaker, adjustable intrinsic connections. An alternative explanation for the late onset of cross-layer map plasticity is that the capacity for plasticity in the superficial OT is simply delayed until the late juvenile phase.

In late juvenile owls, the auditory map in the superficial OT is able to shift relative to the map in the deep OT (Figs. 3-5). The appearance of large-scale, maintained misalignments between superficial and deep OT maps suggests that, at this later age, the normal feedforward connections can lose their dominance over other auditory inputs, specifically adjustable intrinsic inputs. The loss of dominance of the normal feedforward connections could be caused by an increased ability of experience to weaken the functionally maladaptive, normal feedforward connections or to strengthen intrinsic connections, or both. Such an agedependent increase in the relative plasticity of intrinsic versus input projections also occurs in the mammalian neocortex (Singer, 1995; Gilbert, 1998; Stern et al., 2001; Desai et al., 2002).

\section{References}

Binns KE, Salt TE (1998) Experience-dependent changes in the importance of $N$-methyl-D-aspartate (NMDA) receptors for visual transmission in superior colliculus. Brain Res Dev Brain Res 110:241-248.

Brainard MS, Knudsen EI (1993) Experience-dependent plasticity in the inferior colliculus: a site for visual calibration of the neural representation of auditory space in the barn owl. J Neurosci 13:4589-4608.

Brainard MS, Knudsen EI (1995) Dynamics of visually guided auditory plasticity in the optic tectum of the barn owl. J Neurophysiol 73:595-614.

Brainard MS, Knudsen EI (1998) Sensitive periods for visual calibration of the auditory space map in the barn owl optic tectum. J Neurosci 18:3929-3942.

Casagrande VA, Diamond IT (1974) Ablation study of the superior colliculus in the tree shrew (Tupaia glis). J Comp Neurol 156:207-238.

Casagrande VA, Harting JK, Hall WC, Diamond IT, Martin GF (1972) Superior colliculus of the tree shrew: a structural and functional subdivision into superficial and deep layers. Science 177:444-447.

Cohen YE, Knudsen EI (1999) Maps versus clusters: different representations of auditory space in the midbrain and forebrain. Trends Neurosci 22:128-135.

Cook JE, Becker DL (1990) Spontaneous activity as a determinant of axonal connections. Eur J Neurosci 2:162-169.

Daw NW, Fox K, Sato H, Czepita D (1992) Critical period for monocular deprivation in the cat visual cortex. J Neurophysiol 67:197-202.

DeBello WM, Feldman DE, Knudsen EI (2001) Adaptive axonal remodeling in the midbrain auditory space map. J Neurosci 21:3161-3174.

Desai NS, Cudmore RH, Nelson SB, Turrigiano GG (2002) Critical periods for experience-dependent synaptic scaling in visual cortex. Nat Neurosci 5:783-789.

Feldman DE, Knudsen EI (1994) NMDA and non-NMDA glutamate receptors in auditory transmission in the barn owl inferior colliculus. J Neurosci 14:5939-5958.

Feldman DE, Knudsen EI (1997) An anatomical basis for visual calibration of the auditory space map in the barn owl's midbrain. J Neurosci 17:6820-6837.

Gilbert CD (1998) Adult cortical dynamics. Physiol Rev 78:467-485.

Glazewski S, Fox K (1996) Time course of experience-dependent synaptic potentiation and depression in barrel cortex of adolescent rats. J Neurophysiol 75:1714-1729.

GoldJI, Knudsen EI (2000) A site of auditory experience-dependent plasticity in the neural representation of auditory space in the barn owl's inferior colliculus. JNeurosci 20:3469-3486.

Gutfreund Y, Zheng W, Knudsen EI (2002) Gated visual input to the central auditory system. Science 297:1556-1558.

Hyde PS, Knudsen EI (2000) Topographic projection from the optic tectum to the auditory space map in the inferior colliculus of the barn owl. J Comp Neurol 421:146-160.

Hyde PS, Knudsen EI (2002) The optic tectum controls visually guided adaptive plasticity in the owl's auditory space map. Nature 415:73-76.

Jones EG (2000) Cortical and subcortical contributions to activity-dependent plasticity in primate somatosensory cortex. Annu Rev Neurosci 23:1-37.

King AJ, Schnupp JW, Thompson ID (1998) Signals from the superficial layers of the superior colliculus enable the development of the auditory space map in the deeper layers. J Neurosci 18:9394-9408.

Knudsen EI (1982) Auditory and visual maps of space in the optic tectum of the owl. J Neurosci 2:1177-1194.

Knudsen EI (1998) Capacity for plasticity in the adult owl auditory system expanded by juvenile experience. Science 279:1531-1533.

Knudsen EI (1999) Mechanisms of experience-dependent plasticity in the auditory localization pathway of the barn owl. J Comp Physiol 185:305-321.

Knudsen EI (2002) Instructed learning in the auditory localization pathway of the barn owl. Nature 417:322-328.

Knudsen EI, Brainard MS (1991) Visual instruction of the neural map of auditory space in the developing optic tectum. Science 253:85-87.

Knudsen EI, Knudsen PF (1983) Space-mapped auditory projections from the inferior colliculus to the optic tectum in the barn owl (Tyto alba). J Comp Neurol 218:187-196. 
Knudsen EI, Knudsen PF, Esterly SD (1982) Early auditory experience modifies sound localization in barn owls. Nature 295:238-240.

Ramon y Cajal S (1911) Histologie du systeme nerveux de l'homme et des vertebres. Paris: Maloine.

Raymond JL, Lisberger SG, Mauk MD (1996) The cerebellum: a neuronal learning machine? Science 272:1126-1131.

Reh TA, Constantine-Paton M (1985) Eye-specific segregation requires neural activity in three-eyed Rana pipiens. J Neurosci 5:1132-1143.

Reiner A, Karten HJ (1982) Laminar distribution of the cells of origin of the descending pathways in the pigeon (Columba livia). J Comp Neurol 201:165-187.

Singer W (1995) Development and plasticity of cortical processing architectures. Science 270:758-764.

Sprague JM (1991) The role of the superior colliculus in facilitating visual attention and form perception. Proc Natl Acad Sci USA 88: $1286-1290$
Sprague JM, Meikle THJ (1965) The role of the superior colliculus in visually guided behavior. Exp Neurol 11:115-146.

Stein BE, Meredith MA (1993) The merging of the senses. Cambridge, MA: MIT.

Stern EA, Maravall M, Svoboda K (2001) Rapid development and plasticity of layer 2/3 maps in rat barrel cortex in vivo. Neuron 31:305-315.

Tunkl JE, Berkley MA (1977) The role of superior colliculus in vision: visual form discrimination in cats with superior colliculus ablations. J Comp Neurol 176:575-587.

Udin S (1983) Abnormal visual input leads to development of abnormal axon trajectories in frogs. Nature 301:336-338.

Zheng GL, Knudsen EI (1999) Functional selection of adaptive auditory space map by GABA-mediated inhibition. Science 284:962-965.

Zheng W, Knudsen EI (2001) GABAergic inhibition antagonizes adaptive adjustment of the owl's auditory space map during the initial phase of plasticity. J Neurosci 21:4356-4365. 